\title{
miR-132 Over Expression in Osteoarthritis may Regulate the Proliferation and Apoptosis of Fibroblast-like Synoviocytes via Targeting Sirtuin1
}

\author{
L. X. PAN AND W. DING* \\ Department of Orthopedics, Ningbo Medical Center Lihuili Hospital, No. 57, Xingning Road, Ningbo, Zhejiang 315040, China
}

Pan and Ding: Role of miR-132 in osteoarthritis

\begin{abstract}
This present investigation aimed to explore the role and clinical significance of miR-132 in the pathogenesis of osteoarthritis. Twenty osteoarthritis patients and 20 healthy volunteers were enrolled in the present study. The synovial tissues and plasma of the patients and controls were collected and RT-qPCR was performed to determine the expression level of miR-132. In addition the osteoarthritis patient-derived fibroblast-like synoviocytes were isolated, cultured, transfected with miR-132 inhibitors and the proliferation and apoptosis of the cells was determined using MTT assay as well flowcytometry. Finally, the relationship between miR-132 and sirtuin1 in osteoarthritis was evaluated. miR-132 expression was significantly increased in both synovial tissues and plasma of osteoarthritis patients and results of receiver operating characteristic curve showed that both synovial and plasma miR-132 could serve as potential diagnostic biomarker for osteoarthritis with high sensitivity and specificity. Moreover, miR-132 inhibitors significantly inhibited the proliferation and promoted apoptosis of osteoarthritis patients-derived fibroblast-like synoviocytes. Finally, the expression of miR-132 and sirtuin1 was negatively correlated in osteoarthritis and sirtuin1 was confirmed to be a direct target of miR-132. miR-132 was up-regulated in osteoarthritis and miR-132 might promote the development of osteoarthritis through regulating the proliferation and apoptosis of osteoarthritis patients-derived fibroblast-like synoviocytes via targeting sirtuin1.
\end{abstract}

Key words: miR-132, osteoarthritis, fibroblast-like synoviocytes, SIRT1

Osteoarthritis (OA) is a common chronic degenerative disease with high incidence rate ${ }^{[1,2]}$. It mainly occurs in middle-aged and elderly people and the high morbidity and disability rate of the disease can seriously affect the quality of life of affected patients ${ }^{[3]}$. According to the epidemiological investigation in China, the prevalence of OA is about $10 \%$ and this number can reach to $80 \%$ for people over 75 y old $^{[3,4]}$. Unfortunately, at present, the exact pathogenesis of $\mathrm{OA}$ has not been fully elucidated; therefore, exploration of the underlying mechanism of the disease is of great importance to discover new biomarkers and therapeutic targets.

MicroRNAs are a class of endogenous non-coding, single-stranded small RNAs with length of about 20-22 nucleotides. It has been found that they are ubiquitous in multicellular organisms ${ }^{[5,6]}$. Mature microRNAs (miRNA) can post-transcriptionally regulate the gene expression by mediating the silencing target mRNA, which consequentially affected the synthesis of the target proteins ${ }^{[6]}$. In recent years, with the in-depth investigation of miRNAs, it has been found that miRNAs are associated with the development of many human diseases, for example cancers ${ }^{[7,8]}$, autoimmune disease $^{[9,10]}$ and cardiovascular diseases ${ }^{[11]}$.

The regulatory roles of miRNAs in bone and joint diseases, including OA have also been discussed ${ }^{[12-14]}$. In a previous microarray analysis, it was reported that miR-132 was abnormally up-regulated in patients with $\mathrm{OA}^{[15]}$, suggesting that miR-132 might be involved in the pathogenesis of OA. Therefore, in order to confirm the role of miR-132 in osteoarthritis and the possible regulatory mechanism, synovial tissue and plasma samples were collected from patients with clinical OA and the expression of miR-132 using RT-PCR and the

*Address for correspondence

E-mail: dingwei99055@163.com 
effect of miR-132 on the proliferation and apoptosis of fibroblast-like synoviocytes were also investigated. The results of the present work would provide the basis for miRNA to be a potential diagnostic biomarker and a therapeutic target of the disease.

\section{MATERIAL AND METHODS}

\section{Patients and samples:}

In this study, 40 synovial tissues were collected at Ningbo Medical Center Lihuili Hospital. The candidates included 20 patients with osteoarthritis (OA group), 8 males and 12 females, aged between 47-73 y. The diagnosis of OA was based on the diagnostic criteria for osteoarthritis ${ }^{[16]}$ established by the American Rheumatological Society in 1995. Patients with abnormal liver and kidney function, malignant tumours and systemic infections were excluded from this work. There were 20 non-osteoarthritis patients, including 10 males and 10 females, who served as the control group. The synovium in the control group came from patients undergoing amputation due to trauma. Synovial tissue specimens obtained during the operation were immediately frozen in liquid nitrogen and completed within half an hour to reduce the risk of RNA degradation in the tissue. The plasma samples of the patients and healthy controls were also collected. All patients signed informed consent document and this study protocol was approved by the ethical committee of Ningbo Medical Center Lihuili Hospital.

\section{Isolation of the fibroblast-like synoviocytes:}

Fibroblast-like synoviocytes (FS) were isolated from the OA patients. Briefly, the synovial tissue obtained from the OA patients was cut into small pieces under sterile conditions within $2 \mathrm{~h}$, digested by $3.0 \mathrm{~g} / \mathrm{l}$ trypsin (Gibco/Thermo Fisher Scientific, Inc., Waltham, MA, USA) at $37^{\circ}$ for $2 \mathrm{~h}$ and centrifuged for $10 \mathrm{~min}$ at $1000 \mathrm{rpm}$. Then the cells were collected and placed in the medium at $37^{\circ}$. Cells were passaged when the confluence reached $80-90 \%$, and the cells of the 3 to 5 generations were selected for the experiments.

\section{Cell transfection:}

The cell study included 3 groups, the control group, the miR-132 inhibitors and the miR-132 inhibitors negative control group (NC). The miR-132 inhibitors and NC were designed and synthesized by Gene Pharma (Shanghai, China). Transfection was conducted using the Lipofectamine RNAi Max reagent (Invitrogen/ Thermo Fisher Scientific, Inc., Waltham, MA, USA), and the transfection efficiency was confirmed using RTPCR analysis. The effect of miR-132 inhibitors on the behaviors of cells was analysed at $0-48 \mathrm{~h}$.

\section{RT-PCR:}

The total RNA of the synovial tissue, plasma and OA derived FS cells (OAFSs) were isolated by Trizol (Invitrogen) and cDNA were reverse-transcribed and amplified by the Prime Script One-step RT-qPCR kit (Invitrogen) according to the protocols of the manufacturer. The procedure of the RT-qPCR reaction was, $95^{\circ} 10 \mathrm{~min}$; then 50 cycles of $95^{\circ} 10 \mathrm{~s}, 55^{\circ} 10 \mathrm{~s}$, $72^{\circ} 5 \mathrm{~s}, 99^{\circ} 1 \mathrm{~s}, 59^{\circ} 15 \mathrm{~s}$ and $95^{\circ} 1 \mathrm{~s}$. U6 was used as the internal control, and the relative expression of miR-132 was normalized to the expression of U6 by $2^{-\Delta \Delta \mathrm{Ct}}$ method. The primers were synthesized by Genescript (Nanjing, China) and the sequences of the primers were, miR132 forward- 5'-CGCGTAACAGTCTACAGCCA-3' and reverse- 5'-AGTGCAGGGTCCGAGGTATT- 3'; U6 forward- 5'-CTCGCTTCGGCAGCACA-3' and reverse- 5'-AACGCT TCACGAATTTGCGT- 3'.

\section{Cell proliferation assay:}

MTT method was used for the cell proliferation analysis. OAFSs of the control group, miR-132 inhibitors and NC group were treated with 10 ul MTT solution and cultured for $4 \mathrm{~h}$. Then supernatant was discarded and $100 \mu$ dimethyl sulfoxide was added to each well, and OD value at $490 \mathrm{~nm}$ was measured.

\section{Cell apoptosis analysis:}

At $48 \mathrm{~h}$ after transfection, the OAFSs were collected and stained with both PI and Annexin V-FITC reagent using commercially available apoptosis kit (Beyotime, Shanghai, China). The effect of apoptosis rate of the OAFSs was then measured by the BD FACSCalibur flow cytometer (BD Biosciences, San Jose, CA, USA) according to the manufacturer's instructions.

\section{Western blotting:}

The proteins were isolated from the synovial tissue samples and OAFSs with the radioimmunoprecipitation assay buffer (Beyotime, Shanghai, China). The concentration of the proteins in each sample was determined by BCA kit (Beyotime). Then SDS-PAGE was performed to separate the proteins using $10 \%$ gel and the protein on the gel was then transferred to PVDF membranes. Next, the membranes were blocked by $5 \%$ non-fat dry milk and then incubated with the primary antibodies (All purchased from Cell 
Signaling Technology, Inc., Danvers, MA, USA) at $4^{\circ}$ overnight. On day 2 , the membranes were incubated with the secondary antibodies at room temperature for 45 min and then treated by enhanced chemiluminescent substrate (Beyotime) and imaged with the Bio-Rad gel imaging system (Hercules, CA, USA).

\section{Dual Luciferase reporter assay:}

To confirm the direct targeting relationship between miR-132 and SIRT1, dual luciferase reporter assay was performed. Briefly, the wild-type SIRT1 3'-UTR (WT SIRT1-3'UTR) and the mutant SIRT1 3'-UTR (MUT SIRT1) fragments that contained the binding site of miR-132 were synthesized (GenePharma, Shanghai, China), cloned into the commercially available reporter plasmid (p-MIR-reporter plasmid, Thermo Fisher Scientific). Then the plasmids were co-transfected into 293 cells with miR-132 mimics or the NC by Lipofectamine RNAi Max (Invitrogen). After $48 \mathrm{~h}$, the activity of the luciferases was measured by the dualluciferase reporter kit (Beyotime).

\section{Statistical analysis:}

Statistical analysis was performed by Graphad Prism 7.0 (San Diego, USA). The data were expressed as means \pm standard deviation. Comparisons between 2 groups were made using student t-test, and comparisons among three groups were made with ANOVA. Pearson's coefficient analysis was performed for the correlation study and receiver operating characteristic (ROC) curves were used for the evaluation of diagnostic sensitivity and specificity of miR-132. All experiments were repeated for at least 3 times, and $\mathrm{P}<0.05$ indicates statistical significance.

To begin with, RT-PCR analyses were performed to compare the expression level of miR-132 in synovial tissues and plasma of OA patients and healthy controls. It was found that the expression level of miR-132 was significantly higher in the synovial tissues (fig. 1A, $\mathrm{p}<0.05$ ) and plasma (fig. $1 \mathrm{~B}, \mathrm{p}<0.05$ ) of patients with $\mathrm{OA}$ in comparison to healthy controls. Furthermore, results of Pearson correlation analysis showed that there is a strong positive correlation between the expression of miR-132 in synovial tissues and plasma of OA patients (fig. 1C, $r=0.567, \mathrm{p}=0.009$ ).

Moreover ROC curves were drawn to evaluate the ability of synovial tissues and plasma expression of miR-132 to distinguish OA patients from the healthy subjects. Results showed that the AUC for synovial tissues and plasma miR-132 is 0.8625 (fig. 2A), $95 \%$ confidence interval 0.7406 to 0.9844 and 0.8275 (fig. 2B), $95 \%$ confidence interval 0.6993 to 0.9557 , respectively, suggesting that the expression of miR-132 in either synovial tissues or plasma could serve as a diagnostic biomarker for OA.

To further explore the function of miR-132 in the pathogenesis of OA, OAFSs were isolated from OA patients and transfected with the miR-132 inhibitors, and MTT proliferation assay as well as flow cytometry apoptosis assay were conducted. Results of MTT and flow cytometry assays showed that miR-132 inhibitors markedly inhibited proliferation (fig. $3 \mathrm{~A}, \mathrm{p}<0.05$ ) and promoted apoptosis (fig. $3 \mathrm{C}, \mathrm{p}<0.05$ ) of OAFSs in vitro. Furthermore, miR-132 inhibitors also increased the expression of apoptotic protein Bax and decreased the expression of antiapoptotic proteins Bcl-2 (fig. 3B).
A

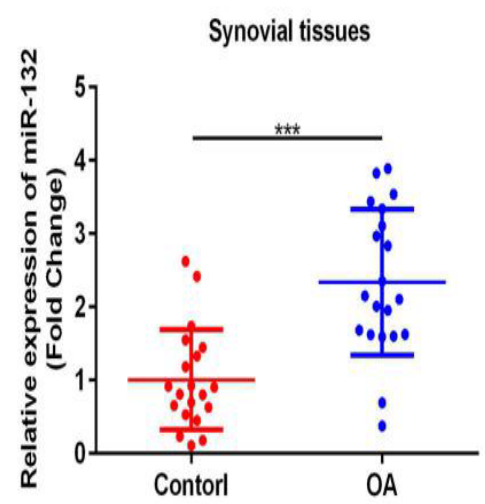

B

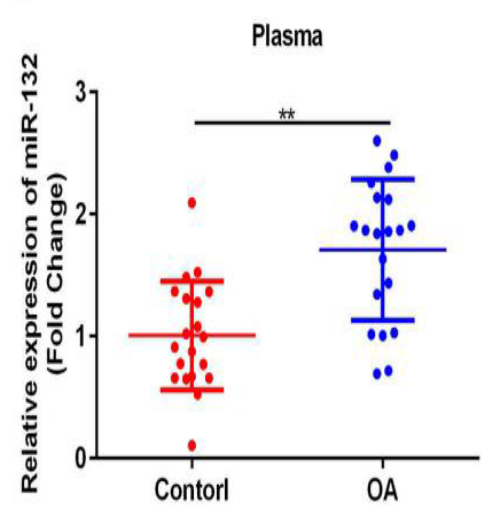

ig. 1: Increased expression of miR-132 in synovial tissues and plasma of patients with $\mathrm{OA}$

A. Comparison of miR-132 expression in synovial tissues of OA patients and healthy controls. B. Comparison of miR132 expression in plasma of $\mathrm{OA}$ patients and healthy controls. C. Correlation between the expression of miR-132 in synovial tissues and plasma of patients with OA. $* * p<0.01, * * * p<0.001$

Special Issue 3, 2020

Indian Journal of Pharmaceutical Sciences
C

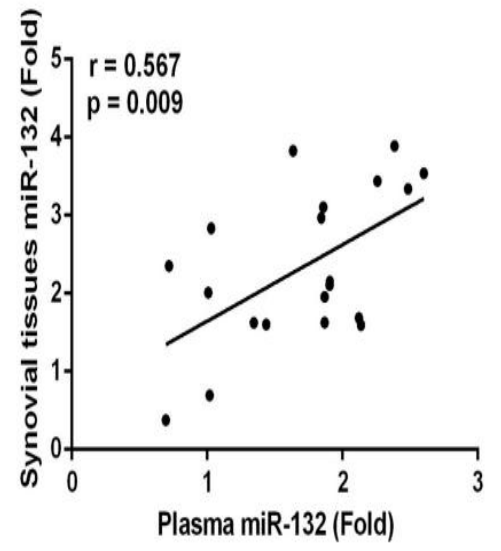




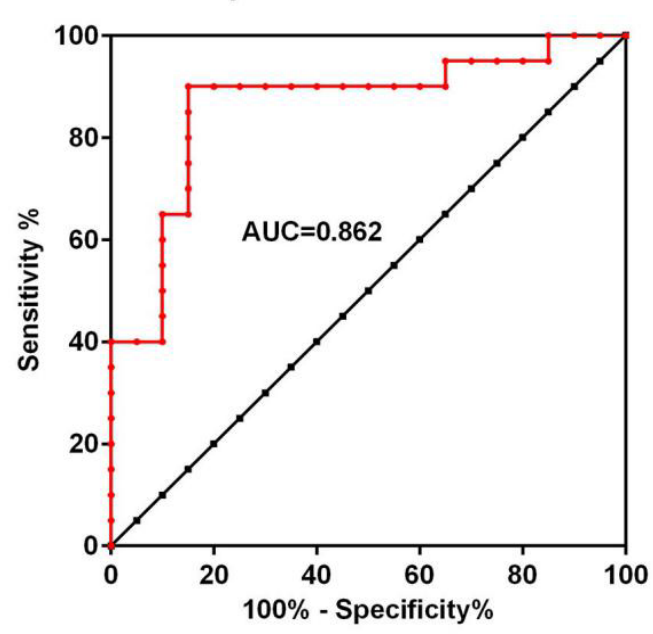

B

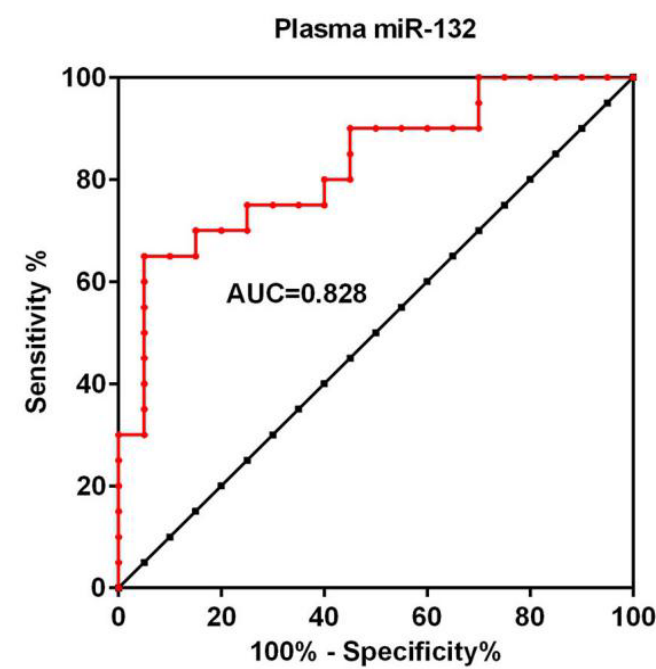

Fig. 2: miR-132 may serve as potential diagnostic biomarker for OA

A. ROC analysis for the expression of miR-132 in synovial tissues to distinguish OA patients from the healthy subjects. B. ROC analysis for the expression of miR-132 in plasma to distinguish OA patients from the healthy subjects.

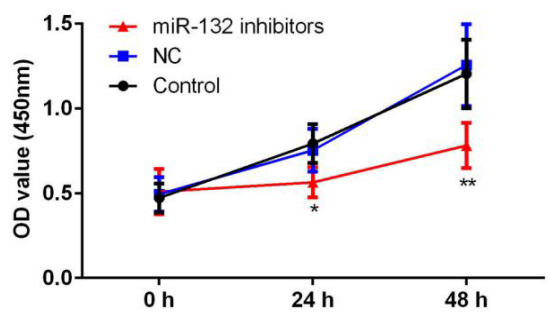

C
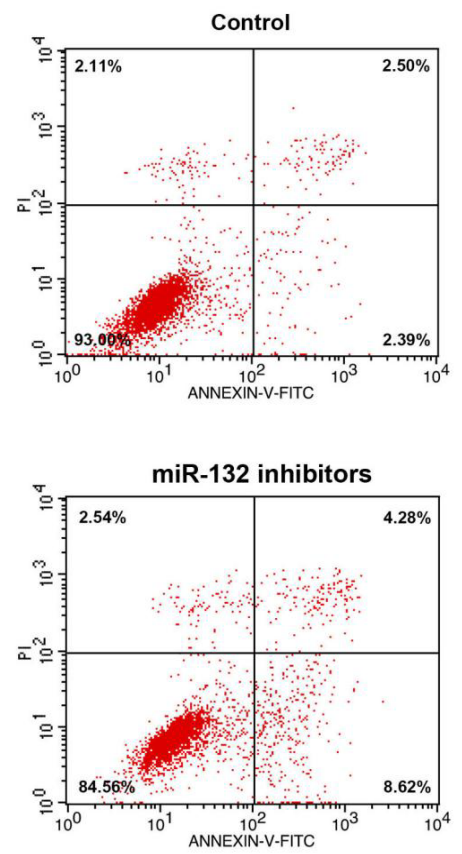

B
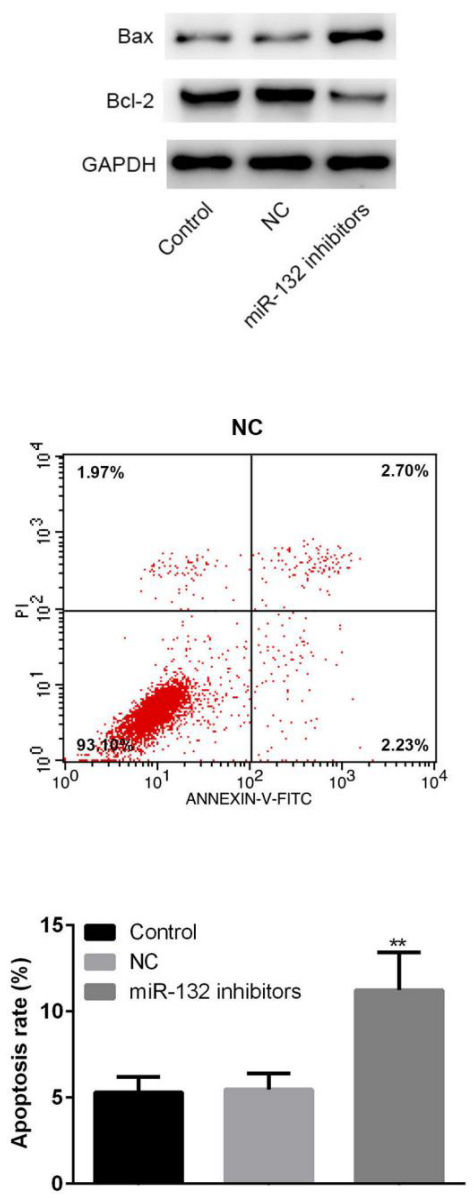

Fig. 3: miR-132 can regulate the proliferation and apoptosis of OAFSs in vitro

A. Effect of miR-132 inhibitor on the proliferation ability of OAFSs in vitro. B. Effect of miR-132 inhibitor on the expression of Bax and Bcl-2 in OAFSs in vitro. C. Effect of miR-132 inhibitor on the apoptosis of OAFSs in vitro. $* \mathbf{p}<0.05, * * \mathbf{p}<0.01$. 
Next, the underlying mechanisms of miR-132 in regulating proliferation and apoptosis of OAFSs were evaluated. First, bioinformatic analysis was performed, which indicated that sirtuin1 (SIRT1) is a target gene of miR-132 (fig. 4A); moreover, the expression of SIRT1 in synovial tissues was evaluated to find that the protein expression of SIRT1 was significantly down-regulated in synovial tissues (fig. 4B and 4C); furthermore, results of correlation analysis showed the expression level of miR-132 and SIRT1 in synovial tissues of OA patients were negatively correlated (fig. $4 \mathrm{D}, \mathrm{r}=-0.607, \mathrm{p}=0.005$ ). Dual luciferase report assay was performed to confirm the targeting relationship between miR-132 and SIRT1. As shown in fig. 5A, miR-132 mimics dramatically inhibited the activity of the luciferase in WT SIRT13'-UTR in OAFSs, while on the other hand, it had no significant effect on the activity of luciferase in MUT SIRT1-3'-UTR treated cells (fig. 5A, p<0.05). Finally, it was found that transfection of miR-132 inhibitor in OAFSs markedly promoted the expression of SIRT1 in OAFSs (fig. 5B).

The present study focused on the roles of miR-132 in $\mathrm{OA}$ and the related mechanism. It was found that miR-132 was up-regulated in both synovial tissues and plasma of OA patients and miR-132 may regulate the proliferation and apoptosis of OAFSs via targeting SIRT1.The aberrant expression of miRNAs in OA
A

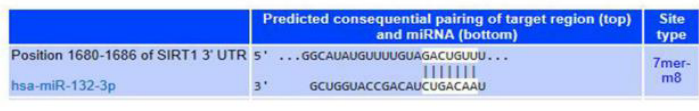

B

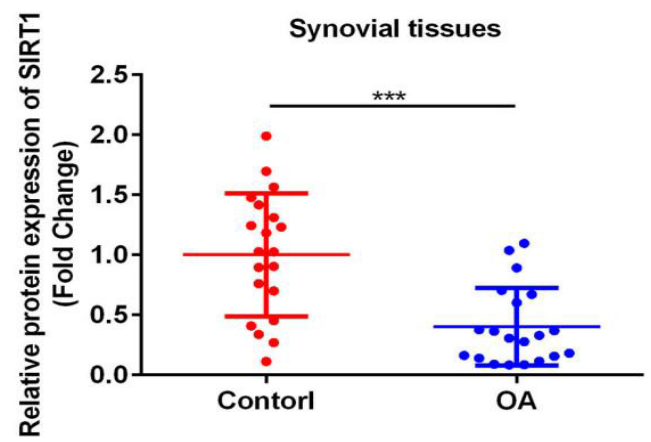

C

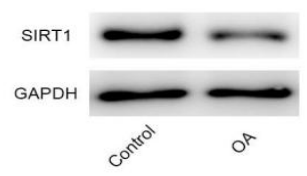

D

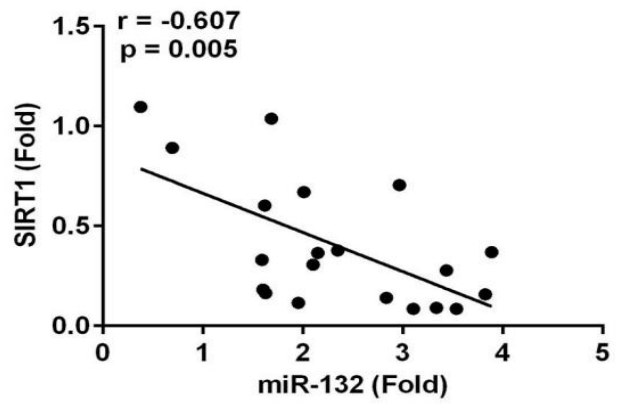

Fig. 4: Decreased expression of SIRT1 in OA

A. The sequences of the paired 3'-UTR region. B. Representative images of the protein expression of SIRT1 in synovial tissues of $\mathrm{OA}$ patients and control. C. Quantified results of the protein expression of SIRT1 in synovial tissues of OA patients and control. D. Correlation between the expression of miR-132 and protein expression of SIRT1 in synovial tissues of OA patients. $* * * \mathbf{p}<\mathbf{0 . 0 0 1}$.

A

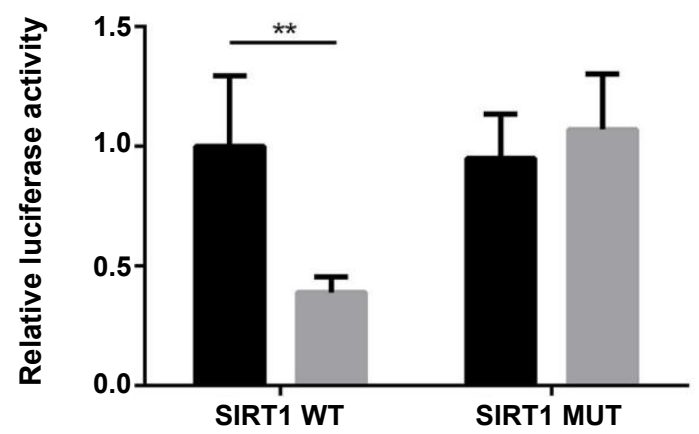

B

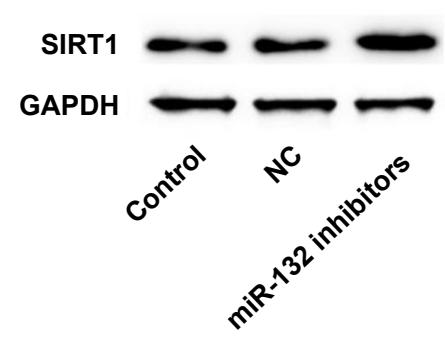

Fig. 5: SIRT1 is a target of miR-132 in OA

A. Results of dual luciferase report assay to confirm the targeting relationship between miR-132 and SIRT1. B. Effect of miR-132 inhibitor on the protein expression of SIRT1 in OAFSs. **p<0.01. (-) control, ( $(-)$ miR-132 inhibitors 
was found in many previous studies. For example, Liu et al reported that miR-211-5p may contribute to the differentiation of chondrocytes by targeting Fibulin- 4 in $\mathrm{OA}^{[17]}$; Yuan et al suggested that miR-214 can alleviate the symptoms of osteoarthritis in rat OA models via regulating the MAPK signalling ${ }^{[18]}$. In Murata et al. work, they performed microarray analysis and found miR-132 was significantly up-regulated in plasma and synovial fluid of the OA patients ${ }^{[15]}$. However, the expression pattern of miR-132 in synovial tissues of OA patients is still unknown. In the present study, we found the expression of miR-132 was significantly increased in synovial tissues of OA patients, which was consistent with Murata et al. finding, suggesting that miR-132 may play important roles in the pathogenesis of OA.

Moreover, results of ROC analysis showed that the expression level of miR-132 in synovial tissues could distinguish the OA patients from the healthy controls. Indeed, these data proposed the possibility of synovial level of miR-132 as a diagnostic marker for $\mathrm{OA}$; however, the difficulty in obtaining the synovial tissues may limit its potential clinical application. Nevertheless, results of previous studies suggested that miRNAs can be released by cells in to circulation and interestingly, unlike most of the protein biomarkers, those circulating miRNAs are stable in the peripheral blood $^{[19,20]}$. Therefore, numerous previous studies have proposed the roles of circulating miRNAs as potential diagnostic biomarkers for different diseases ${ }^{[19-22]}$, including $\mathrm{OA}^{[23,24]}$. In Murata's sequencing analysis, miR-132 was also found to be up-regulated in plasma of the OA patients, however, the results have not been verified by RT-PCR analysis with larger sample size and whether miR-132 can serve as a diagnostic biomarker is still unclear. In the present study, miR-132 was found to be significantly increased in plasma of the OA patients, which confirmed Murata's finding, and interestingly, the expression of miR-132 in the synovial tissue and plasma of the OA patients were positively correlated; furthermore, ROC analysis showed the AUC of circulating miR-132 for the diagnosis of OA was 0.8257 , which was similar to the AUC of synovial miR-132 (0.8625). Taken together, these results suggested that miR-132 was also elevated in plasma of OA patients, and the circulating miR-132 may serve as a potential biomarker for the early diagnosis of OA.

OAFSs have been reported to play a key role in the development of $\mathrm{OA}^{[25,26]}$. Over-proliferation of OAFSs could lead to long-term inflammatory condition and finally lead to the degradation of the cartilage ${ }^{[27-29]}$. In the present study, miR-132 inhibitor was found to inhibit the proliferation and promoted the apoptosis of OAFSs, suggesting that miR-132 may be involved in the pathogenesis of $\mathrm{OA}$ via regulating the proliferation of OAFSs.

miRNAs were known to exert their functions via inhibiting their targets. Sirtuin1 (SIRT1) belongs to the sirtuin family which are a group of NAD+-dependent protein deacetylases ${ }^{[30,31]}$. Results of previous studies suggested that it was down regulated in OA, and overexpression of SIRT1 may play protective role by inhibiting the secretion of inflammatory cytokines and degradation of the cartilage ${ }^{[32,33]}$. In the present study, we used bioinformatic methods and SIRT1 has been predicted as a target of miR-132; moreover, the protein expression of SIRT1 was down-regulated in synovial tissues of OA patients and the expression of miR-132 and SIRT1 was negatively correlated; furthermore, we confirmed the directing targeting relationship between miR-132 and SIRT1 in OA by luciferase reporter assay and WB. Taken together, our data suggested that overexpression of miR-132 in OA may lead to the decreased expression of SIRT1, which consequentially lead to the over-proliferation of OAFSs and contribute to the development of the disease.

In conclusion, it was found that miR-132 was upregulated in both synovial tissues and plasma of OA patients and miR-132 could regulate proliferation and apoptosis of OAFSs via targeting SIRT1. The results of the present study proposed the potential application of miR-132 as a diagnostic marker and therapeutic target for OA.

\section{Conflict of interest:}

All authors report no conflicts of interest in this work.

\section{REFERENCES}

1. Allas L, Rochoux Q, Leclercq S, Boumediene K, Bauge C. Development of a simple osteoarthritis model useful to predict in vitro the anti-hypertrophic action of drugs. Lab Invest 2020;100:64-71.

2. Dai X, Ding W, Li H, Xu P, Huang Z, Zhu W, et al. Associations of Serum Lipids and Deep Venous Thrombosis Risk After Total Knee Arthroplasty in Patients With Primary Knee Osteoarthritis. Int J Low Extrem Wounds 2020;19:51-6.

3. Gui Q, Zhang X, Liu L, Zhao F, Cheng W, Zhang Y. Costutility analysis of total knee arthroplasty for osteoarthritis in a regional medical centre in China. Health Econ Rev 2019;9:15.

4. Wang X, Ning Y, Zhang P, Yang L, Li C, Zhou R, et al. Biological Analysis of Gene Expression and Clinical Variables Suggest FZD1 as a Novel Biomarker for Patients with Kashin- 
Beck Disease, an Endemic Osteoarthritis in China. Dis Markers 2019;3736198.

5. Ye J, Xu M, Tian X, Cai S, Zeng S. Research advances in the detection of miRNA. J Pharm Anal 2019;9:217-26.

6. Zhang Y, Yang C, Yang S, Guo Z. MiRNA-27a decreases ultraviolet B irradiation-induced cell damage. J Cell Biochem 2019;121:1032-8.

7. Yin XH, Jin YH, Cao Y, Wong Y, Weng H, Sun C. Development of a 21-miRNA signature Associated with the prognosis of patients with bladder cancer. Front Oncol 2019;9:729.

8. Zouari M, Campuzano S, Pingarron JM, Raouafi N. Amperometric Biosensing of miRNA-21 in Serum and Cancer Cells at Nanostructured Platforms Using Anti-DNA-RNA Hybrid Antibodies. ACS Omega 2018;3:8923-31.

9. Kim J, Kim DY, Heo HR, Choi SS, Hong SH, Kim WJ. Role of miRNA-181a-2-3p in cadmium-induced inflammatory responses of human bronchial epithelial cells. J Thorac Dis 2019;11:3055-69.

10. Zeh N, Schneider H, Mathias S, Raab N, Kleemann M, Schmidt-Hertel S, et al. Human CAP cells represent a novel source for functional, miRNA-loaded exosome production. PLoS One 2019; 14:e0221679.

11. Liao J, Wang J, Liu Y, Li J, Duan L. Transcriptome sequencing of IncRNA, miRNA, mRNA and interaction network constructing in coronary heart disease. BMC Med Genomics 2019; $12: 124$.

12. Cai C, Min S, Yan B, Liu W, Yang X, Li L, et al. MiR-27a promotes the autophagy and apoptosis of IL-1beta treatedarticular chondrocytes in osteoarthritis through PI3K/AKT/ mTOR signaling. Aging 2019;11:6371.

13. Zhao $\mathrm{H}$ and Gong N. miR-20a regulates inflammatory in osteoarthritis by targeting the IkappaBbeta and regulates NKkappaB signaling pathway activation. Biochem Biophys Res Commun, 2019;518:632-7.

14. Cao J, Liu Z, Zhang L, Li J. miR-940 regulates the inflammatory response of chondrocytes by targeting MyD88 in osteoarthritis. Mol Cell Biochem 2019;461:183-93.

15. Murata K, Yoshitomi H, Tanida S, Ishikawa M, Nishitani K, Ito $\mathrm{H}$, et al. Plasma and synovial fluid microRNAs as potential biomarkers of rheumatoid arthritis and osteoarthritis. Arthritis Res Ther 2010;12:R86.

16. Murphy WA, Altman RD. Updated osteoarthritis reference standard. J Rheumatol Suppl 1995;43:56-59.

17. Liu H, Luo J. MiR-211-5p contributes to chondrocyte differentiation by suppressing Fibulin-4 expression to play a role in osteoarthritis. J Biochem 2019;166:495-502.

18. Yuan L, Chang X, Yao W, He H, Tang Z, Wu J. MiR-214 inhibits knee osteoarthritis in rats through MAPK signaling pathway. Panminerva Med 2019.

19. Swellam M, Ezz El Arab L, Al-Posttany AS, S BS. Clinical impact of circulating oncogenic MiRNA-221 and MiRNA-222 in glioblastoma multiform. J Neurooncol 2019;144:545-51.

20. Hetta HF, Zahran AM, El-Mahdy RI, Nabil EE, Esmaeel HM, Alkady OA, et al. Assessment of Circulating miRNA-17 and miRNA-222 Expression Profiles as Non-Invasive Biomarkers in Egyptian Patients with Non-Small-Cell Lung Cancer. Asian Pac J Cancer Prev 2019;20:1927-33.

21. Kiyosawa N, Watanabe K, Toyama K, Ishizuka H. Circulating miRNA Signature as a Potential Biomarker for the Prediction of Analgesic Efficacy of Hydromorphone. Int J Mol Sci 2019;20:1665.

22. Nakashima H, Yoshida R, Hirosue A, Kawahara K, Sakata J,
Arita $\mathrm{H}$, et al. Circulating miRNA-1290 as a potential biomarker for response to chemo radiotherapy and prognosis of patients with advanced oral squamous cell carcinoma: A single-centre retrospective study. Tumour Biol 2019;41:1010428319826853.

23. Dou X, Zhang Z, Wang S, Zhou X. Combined use of Serum miR-338-3p, Cartilage Oligomeric Matrix Protein and Chondroitin Sulfate-846 in the Early Diagnosis of Knee Osteoarthritis. Clin Lab 2019;65.

24. Ntoumou E, Tzetis M, Braoudaki M, Lambrou G, Poulou M, Malizos $\mathrm{K}$, et al. Serum microRNA array analysis identifies miR-140-3p, miR-33b-3p and miR-671-3p as potential osteoarthritis biomarkers involved in metabolic processes. Clin Epigenetics 2017;9:127.

25. Machado CR, Resende GG, Macedo RB, Branco AS, Kakehasi AM, Andrade MV. Fibroblast-like synoviocytes from fluid and synovial membrane from primary osteoarthritis demonstrate similar production of interleukin 6 , and metalloproteinases 1 and 3. Clin Exp Rheumatol 2019;37:306-9.

26. Cai P, Jiang T, Li B, Qin X, Lu Z, Le Y, et al. Comparison of rheumatoid arthritis (RA) and osteoarthritis (OA) based on microarray profiles of human joint fibroblast-like synoviocytes. Cell Biochem Funct 2019;37:31-41.

27. Pan L, Zhang Y, Chen N, Yang L. Icariin Regulates Cellular Functions and Gene Expression of Osteoarthritis PatientDerived Human Fibroblast-Like Synoviocytes. Int J Mol Sci 2017; 18:2656.

28. Ding X, Zhang Y, Huang Y, Liu S, Lu H, Sun T. Cadherin-11 involves in synovitis and increases the migratory and invasive capacity of fibroblast-like synoviocytes of osteoarthritis. Int Immunopharmacol 2015;26:153-61.

29. Yang CR, Shih KS, Liou JP, Wu YW, Hsieh IN, Lee HY, et al. Denbinobin upregulates miR-146a expression and attenuates IL-1beta-induced upregulation of ICAM-1 and VCAM-1 expressions in osteoarthritis fibroblast-like synoviocytes. J Mol Med 2014;92:1147-58.

30. Feng K, Ge Y, Chen Z, Li X, Liu Z, Li X, et al. Curcumin Inhibits the PERK-eIF2alpha-CHOP Pathway through Promoting SIRT1 Expression in Oxidative Stress-induced Rat Chondrocytes and Ameliorates Osteoarthritis Progression in a Rat Model. Oxid Med Cell Longev 2019;8574386.

31. Feng K, Chen Z, Pengcheng L, Zhang S, Wang X. Quercetin attenuates oxidative stress-induced apoptosis via SIRT1/ AMPK-mediated inhibition of ER stress in rat chondrocytes and prevents the progression of osteoarthritis in a rat model. $\mathrm{J}$ Cell Physiol 2019;234:18192-205.

32. Sun W, Li Y, Wei S. miR-4262 regulates chondrocyte viability, apoptosis, autophagy by targeting SIRT1 and activating PI3K/ AKT/mTOR signalling pathway in rats with osteoarthritis. Exp Ther Med 2018;15:1119-28.

33. Liu S, Yang H, Hu B, Zhang M. Sirtl regulates apoptosis and extracellular matrix degradation in resveratrol-treated osteoarthritis chondrocytes via the Wnt/beta-catenin signaling pathways. Exp Ther Med 2017;14:5057-62.

This is an open access article distributed under the terms of the Creative Commons Attribution-NonCommercial-ShareAlike 3.0 License, which allows others to remix, tweak, and build upon the work non-commercially, as long as the author is credited and the new creations are licensed under the identical terms

This article was originally published in a special issue, "Biomedical applications in Pharmaceutical Sciences" Indian J Pharm Sci 2020:82(2)Spl issue3;142-148 